TITLE:

\title{
ADVANCEMENT OF THE SCALING FFAG ACCELERATOR FOR ADSR, PULSED-SNS AND MUON ACCELERATION
}

$\operatorname{AUTHOR}(S)$ :

MORI, YOSHIHARU

\section{CITATION:}

MORI, YOSHIHARU. ADVANCEMENT OF THE SCALING FFAG ACCELERATOR FOR ADSR, PULSED-SNS AND MUON ACCELERATION. International Journal of Modern Physics A 2011, 26(10-11): 1744-1752

ISSUE DATE:

2011-04

URL:

http://hdl.handle.net/2433/141913

\section{RIGHT:}

(C) 2011 World Scientific Publishing Co.; This is not the published version. Please cite only the published version.; この論文は出版社版で ありません。引用の際には出版社版をご確認ご利用ください。 


\title{
Advancement of the scaling FFAG Accelerator for ADSR, pulsed-SNS and muon acceleration
}

\author{
YOSHIHARU MORI \\ Kyoto University, Research Rector Institute \\ Kumatori, Osaka 590-0494, Japan \\ mori@rri.kyoto-u.ac.jp \\ Received Day Month Year \\ Revised Day Month Year
}

Progress in the study of beam optics and acceleration of the scaling FFAG accelerator has been presented in this paper.

Keywords: FFAG; zero-chromaiticiy.

PACS numbers: 11.25 .Hf, $123.1 \mathrm{~K}$

\section{Introduction}

Production of intense neutrons is essential for accelerator driven sub-critical reactors (ADSR) and pulse spallation neutron sources (SNS). The neutron yield increases very abruptly up to the proton energy of about $1 \mathrm{GeV}$. Compared with more neutron yield at $100 \mathrm{MeV}$, more than two order of magnitude large neutrons can be generated at $1 \mathrm{GeV}$. It should be also noted that in such high energy region, nuclear cascade interaction (hadron shower reaction) is a dominant process and the mean free path for heavy target material such as Ta in this reaction is about $15 \mathrm{~cm}$, which means the target thickness of about $50 \mathrm{~cm}$ can be enough. Thus, from the point of view of neutron yield, higher energy is better.

One beam specification requested of the proton beam for ADSR and pulsed SNS is its time structure. The pulsed SNS requires a short pulse $(<0.5 \mu \mathrm{sec})$ and relatively low repetition proton beam. On the other hand, the beam time structure for the ADSR is rather modest and a relatively higher repetition rate (> $100 \mathrm{~Hz}$ ) is favorable to avoid thermal shock on the target. The types of accelerator which could satisfy both these requirements simultaneously are a proton linear accelerator (LINAC)/proton storage ring(PSR) complex, or a fixed field alternating gradient(FFAG) accelerator. Neither a cyclotron or synchrotron can be used. A LINAC/PSR complex is a straightforward solution but very expensive. On the other hand, a high intensity FFAG accelerator needs more research and development.

In muon acceleration, the FFAG accelerator is useful because of its capabilities of strong beam focusing and very fast acceleration, however, it requires more 
studies especially on the advanced schemes of fast beam acceleration and beam manipulation for injection/extraction with relatively large beam emittance.

In this paper, advancement of the FFAG accelerator which could open the feasibility and capability of the FFAG accelerator for ADSR and pulsed SNS, and also for muon acceleration are discussed.

\section{Beam focusing and acceleration in FFAG accelerator}

The idea of the FFAG accelerator was originally by Ohkawa in $1953^{1}$ and by Symon and Kolomenskii, independently. Electron models of the FFAG accelerator were built by Kerst, Cole and Symon at MURA in the early 1960s ${ }^{2}$. However, since then, no FFAG proton accelerator has been built until recently. A proton FFAG accelerator, in particular, has some severe technical difficulties such as its complicated configuration of magnetic field and difficulty of $\mathrm{rf}$ acceleration. In 2000, the world's first proton FFAG accelerator (POP-FFAG) was demonstrated using a novel broad-band RF cavity with high permeability MA(magnetic alloy) cores at KEK ${ }^{3}$. Following this success, it has been recognized that FFAGs have large advantages in rapid acceleration with large momentum acceptance, which are useful both for muon accelerators and for high power proton drivers. Since then, intensive studies and discussions have been carried out, and various projects for the development of FFAGs have started for many applications at many institutes.

The FFAG accelerator has distinctive features compared with other types of accelerators in beam focusing and acceleration. In transverse beam focusing, two types of beam focusing can be categorized: scaling and non-scaling. The scaling type of FFAG accelerator has non-chromatic beam optics in which a large amount of non-linear field is essential. Fields are linear in the non-scaling type of FFAG accelerator. However, a number of betatron resonances have to be crossed during acceleration. As for beam acceleration, various schemes with new ideas such as a broad-band MA rf cavity, stationary bucket acceleration, serpentine acceleration and harmonic number jump acceleration have been studied intensively and some of them have been realized.

\subsection{Beam focusing}

\subsubsection{Scaling}

In the scaling type of FFAG accelerator, each beam orbit for different beam momentum has similarity in shape (curvature), and zero chromaticity in the beam optics is realized. Thus, the betatron tunes for both horizontal and vertical directions keep constant during beam acceleration without any problems caused by resonance crossing. The transverse linearized betatron equation of motion can be expressed by the following equation.

$$
\frac{d^{2} X}{d s^{2}}+K(s) X=0
$$


where $\mathrm{X}$ is the transverse displacement of the particle and $\mathrm{s}$ is the distance along the reference trajectory, and $\mathrm{K}(\mathrm{s})$ can be given for horizontal $(\mathrm{x})$ and vertical $(\mathrm{z})$ directions by,

$$
\begin{aligned}
& K_{x}=\frac{1-n(s)}{\rho^{2}(s)} \\
& K_{z}=-\frac{n(s)}{\rho^{2}(s)},
\end{aligned}
$$

respectively. Here $\rho$ is a orbit curvature and $\mathrm{n}$ is a field index defined with magnetic field strength as ,

$$
n=-\frac{\rho}{B}\left(\frac{d B}{d X}\right)
$$

The scaling condition requires that the equation of motion of eq.(1) is independent of momentum both for horizontal and vertical directions.

In cylindrical coordinates, it can be shown analytically that the configuration of the magnetic field at the median plane can be expressed with a geometrical field index $\mathrm{k}$ and a reference orbit radius $\mathrm{r}_{0}$ in the following equation including azimuthal direction ${ }^{4}$.

$$
B(r, \theta)=B_{0}\left(\frac{r}{r_{0}}\right)^{k} \mathcal{F}\left(\theta-\zeta \ln \frac{r}{r_{0}}\right)
$$

where $\zeta=\tan \xi$, and $\xi$ is a spiral angle of the magnet in the azimuth plane. Accordingly, two schemes for beam focusing are invoked by this magnetic field configuration: one is radial sector focusing and the other spiral sector focusing. Radial sector focusing uses a combination of positive and negative bending magnets to make strong beam focusing with a FODO lattice configuration. In spiral sector focusing, edge focusing is used efficiently.

\subsubsection{Non-scaling}

The non-scaling type of FFAG accelerator has a basically simple structure composed of linear optical elements such as dipole and quadrupole magnets. The transverse betatron tune, however, is not constant during acceleration and fast acceleration is essential in the non-scaling type of FFAG accelerator to avoid beam losses caused by resonance crossing. To ensure this, the RF cavity phases are fixed for beam acceleration and the range of times-of-flight over the energy range of the accelerator is minimized when the path length difference has a parabolic dependence on beam momentum ${ }^{5}$. Based on the concept of linear non-scaling FFAG accelerators, some variations such as isochronous or semi-achromatic lattice ${ }^{6,7}$ including small nonlinear elements(semi-scaling) have also been proposed. 


\subsubsection{Advances in scaling FFAG optics}

The scaling law of the FFAG accelerator to satisfy the zero chromaticity is applied to circular accelerator as described above and each cell provides a total bending angle of a ring. If a scaling FFAG unit cell with straight section where a overall bending angle is zero is created, insertion/matching optics with straight sections can be cooperated to the ordinary FFAG ring. The scaling condition for the straight section unit cell, i.e. a same phase advance per cell at every energy, leads to a different field law as shown below.

$$
\begin{gathered}
\frac{d\left(\frac{1}{\rho^{2}}\right)}{d p}=0 \\
\frac{d\left(K \rho^{2}\right)}{d p}=0
\end{gathered}
$$

Geometrical similarity is given by $\rho=$ const. and it leads to a solution for the magnetic field configuration ${ }^{8}$.

$$
B=B_{0} e^{m\left(X-X_{0}\right)} \mathcal{F}\left(Y-X \tan \xi_{s}\right) .
$$

Here,

$$
m=\frac{1}{B} \frac{d B}{d X} .
$$

Applying this scaling field law to the FFAG straight section, insertion/matching optics adding to the ordinary scaling FFAG rings have been examined for various applications ${ }^{9,10}$. Figure 1 shows an example of the scaling FFAG straight sections and the dispersion suppressing insertion ${ }^{10}$. Schematic diagram of the scaling FFAG ring with the dispersion suppressing insertion and straight section is presented in Fig. 2.

\subsection{Acceleration}

One of the most distinctive features in FFAG accelerators compared with ordinary strong focusing synchrotrons is that very fast acceleration is possible because the magnetic field is static. Accordingly, various new RF acceleration schemes, which are adequate both for scaling and non-scaling FFAG accelerators, have been proposed for fast acceleration, and some of them have already been realized.

\subsubsection{Broad-band RF cavity with MA cores}

Broad-band RF cavities with high permeability magnetic alloy (MA) cores have been used mostly in the scaling type of proton FFAG accelerators such as the POPFFAG developed at KEK. No frequency tuning synchronized with beam revolution 


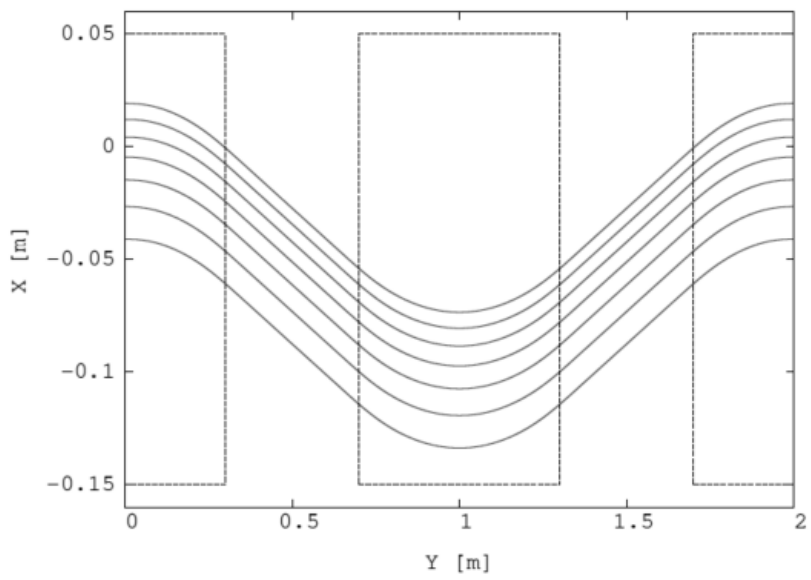

\section{CELL $1 \quad$ CELL 2}

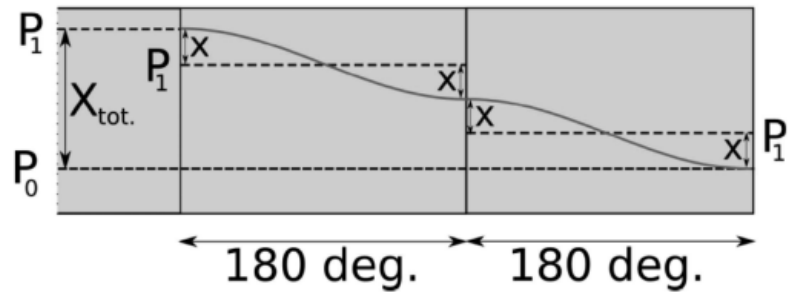

Fig. 1. Scaling FFAG straight line and the dispersion suppressing insertion.

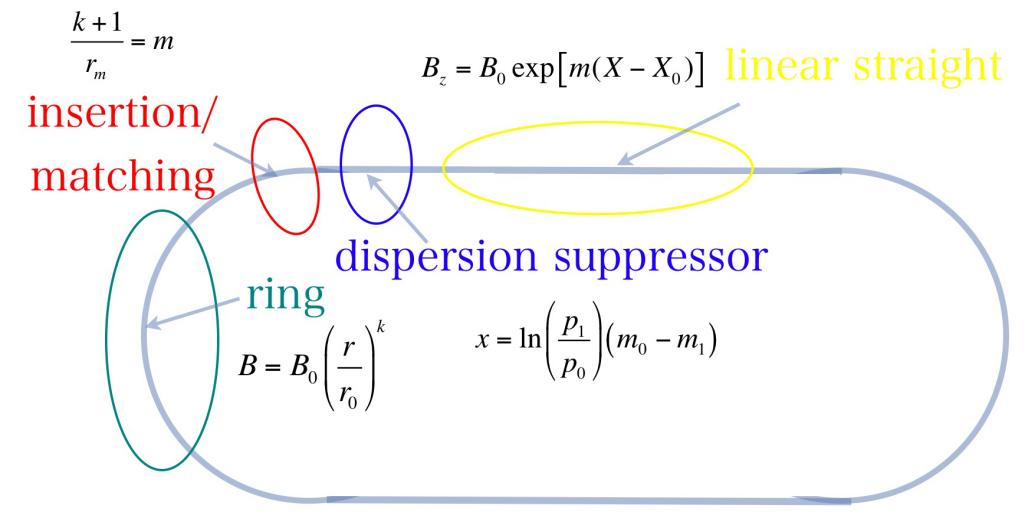

Fig. 2. Schematic diagram of scaling FFAG ring with linear straight section and the dispersion suppressing insertion. 
is needed and a very rapid acceleration cycle of several $100 \mathrm{~Hz}$ to $1 \mathrm{kHz}$ becomes possible. However, above that rate, the required rf voltage becomes rather high, which is difficult to be obtained with this type of rf cavity.

\subsubsection{Stationary $R F$ bucket acceleration}

In longitudinal phase space, the RF bucket of the scaling type of FFAG accelerator for relativistic particles such as high energy muons is not distorted for large momentum range because the momentum compaction keeps constant and has no momentum dependence. If the RF voltage is large enough, the particles can be accelerated in a stationary RF bucket after a half period of synchrotron oscillation ${ }^{11}$.

\subsubsection{Serpentine acceleration}

In the non-scaling FFAG accelerator, a fixed frequency RF system is installed and acceleration out of longitudinal buckets is possible for relativistic particles when the path length difference has a parabolic dependence on momentum as described above. The revolution time (time-of-flight per turn) changes and has a minimum during beam acceleration ${ }^{12}$.

In the scaling FFAG accelerator, a similar acceleration scheme with a fixed frequency RF is possible where the beam is accelerated passing through the transition energy where the time-of-flight per turn is a minimum. The longitudinal Hamiltonian in the case of scaling FFAG accelerator is analytically obtained as,

$$
\frac{H}{m_{0} c^{2} f_{s}}=2 \pi\left[-\frac{A}{2(1-\lambda)}\left(\gamma^{2}-1\right)^{1-\lambda}+\gamma\right]+\frac{e V h}{m_{0} c^{2}} \cos \phi .
$$

Here,

$$
\begin{gathered}
\lambda=\frac{k}{2(k+1)} \\
A=\frac{\left(\gamma_{s}^{2}-1\right)^{\lambda}}{\gamma_{s}}
\end{gathered}
$$

Figure 3 shows an example of the phase space contour for the longitudinal motion of the scaling FFAG accelerator ${ }^{13}$. As can be seen from this figure, a serpentine path through the transition energy exists and beam acceleration for non-relativistic particles can be possible with this scheme in the scaling FFAG accelerator.

\subsubsection{Harmonic number jump}

In FFAG accelerators, the transverse beam positions change during acceleration. If the energy gain varies with transverse position to achieve an integer change in the harmonic number with each turn, the beam can be accelerated with a fixed frequency RF system ${ }^{14}$. The difficulty of this scheme is how to vary the energy 


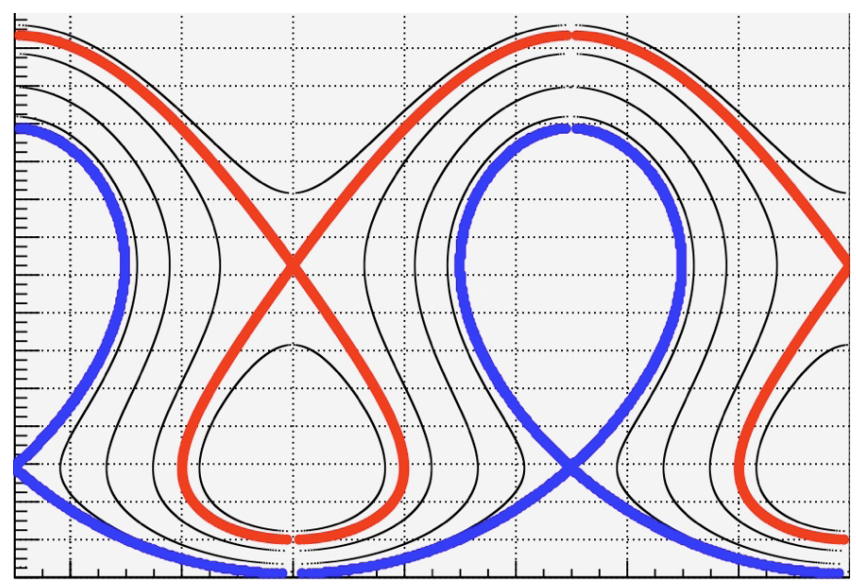

Fig. 3. Longitudinal Hamiltonian contour for serpentine acceleration in scaling FFAG accelerator.

gain properly and ideas such as a position dependent RF voltage configuration have been proposed to overcome this difficulty.

\section{FFAG for high intensity proton accelerator}

,A proton driver for producing an order of magnitude more intense secondary particles such as neutrons, muons, mesons etc. compared with the present HIPA such as J-PARC, SNS and ISIS requires the next generation of high intensity proton accelerator (HIPA). The beam energy of next generation HIPAs should be more than $1 \mathrm{GeV}$ because the nuclear interaction generating hadron shower can be very efficient there and the beam power of more than 10MW is now under discussion. Requirements for the time structure of the beam from next generation HIPAs, however, have many varieties which depend on each application. The neutrino factory/muon collider requires a very short beam bunch (nsec) and low repetition rate operation $(10 \mathrm{~Hz})$. On the other hand, the ADSR requires a very large repetition rate of more than $\mathrm{kHz}$ or even $\mathrm{cw}$ operation. The next generation HIPAs should satisfy these various requirements as much as possible.

The types of accelerator suitable for next generation of HIPA, from this point of view, are limited. Ordinary synchrotron and cyclotron can be excluded. The former is unsuited for high repetition operation and the latter for short bunched and high peak intensity pulse operation. The candidates of the next generation HIPA could be a superconducting LINAC/PSR complex, and a FFAG.proton accelerator. The FFAG accelerator could be operated in various repetition pulsed modes and even in cw mode as described in the previous section. When the beam repetition is less than $1 \mathrm{kHz}$, the broad-band $\mathrm{RF}$ system as described in the previous section can be used for either scaling or non-scaling FFAGs. However, in order to realize cw operation, a fixed-frequency RF acceleration scheme is essential and, in particular, for the proton driver where the particle velocity is still non-relativistic, a scaling 


\begin{tabular}{lr}
\hline Item & value \\
\hline Average radius(m) & 15 \\
Field index & 3 \\
Injection energy & $300 \mathrm{MeV}$ \\
Extraction energy & $2.2 \mathrm{GeV}$ \\
RF voltage per turn $(\mathrm{h}=1)$ & $38 \mathrm{MV}$ \\
\hline
\end{tabular}

FFAG accelerator with serpentine acceleration seems to be a good candidate. Table 1 presents an example of the parameters of a proton driver based on a scaling FFAG accelerator using serpentine beam acceleration shown in Fig. 4.

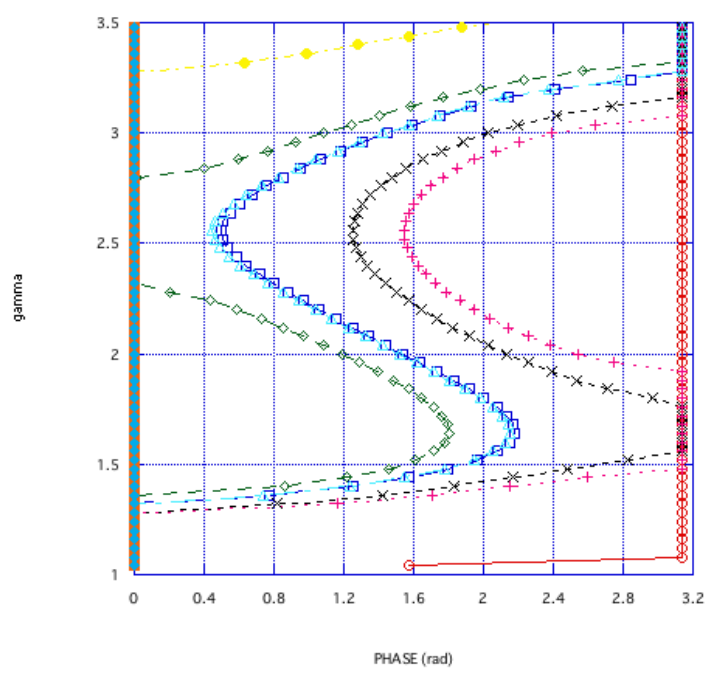

Fig. 4. Serpentine acceleration for $\operatorname{HIPA}(0.3-2.2 \mathrm{GeV})$ with scaling FFAG accelerator.

In KURRI, the present $150 \mathrm{MeV}$ proton FFAG accelerator is used for a basic experimental study of ADSR with Kyoto University Critical Assembly(KUCA). The machine will be upgraded soon to a the beam intensity of up to 5-10 $\mu \mathrm{A}$ with a repetition rate of $30 \mathrm{~Hz}$ using a $\mathrm{H}^{-}$proton linac which exists already. The beam energy energy upgrade could be realized with an additional spiral FFAG having a diameter of about $16 \mathrm{~m}$. For the high power ADSR experiment, the beam repetition is to be increased to more than $300 \mathrm{~Hz}$ with additional $\mathrm{RF}$ acceleration systems for both $150 \mathrm{MeV}$ and $700 \mathrm{MeV}$ rings, and the thermal output power of the ADSR could become more than $5 \mathrm{MW}$. 


\section{Summary}

Beam focusing and acceleration in the Fixed Field Alterenating Gradient Accelerator (FFAG) have been briefly described and a possible concept of FFAG accelerator for next generation High Intensity Proton Accelerator for ADSR and pulsed SNS discussed. The future upgrade scenario and design of the FFAG upgrade at KURRI for ADSR and pulsed SNS, where the beam energy will be more than $700 \mathrm{MeV}$ and the beam power will be $5 \mathrm{~kW}$ with $30 \mathrm{~Hz}$ repetition rate for pulsed SNS and $50 \mathrm{~kW}$ with $300 \mathrm{~Hz}$ rate for high power ADSR, have also been described.

\section{References}

1. T.Ohkawa, Proc. annual meeting of JPS,(1953).

2. K.R.Symon et al., Phys. Rev. 103,1837(1956).

3. Y.Mori, Proc. EPAC1998, 289(2000).

4. A.A.Kolomenski, "Theory of Circular Accelerators", 340(1966).

5. D.Trbojevic, Proc. of the International Workshop on FFAG Accelerators (FFAG05), KURRI, Osaka, Japan(2005).

6. G.Rees, Proc. of the International Workshop on FFAG Accelerators (FFAG04), KEK, Tsukuba, Japan, 77( 2004).

7. A.G. Ruggeiero, Proc. of the International Workshop on FFAG Accelerators (FFAG05), KURRI, Osaka, Japan, 47(2005).

8. JB.Lagrange, T. Planche and Y.Mori, Proc. of FFAG09, FNAL, Chicago, (2009).

9. Y.Mori, International Workshop on FFAG Accelerators(FFAG09) ,FNAL, Chicago, USA(2009).

10. T.Planche and Y.Mori, Proc. of PAC09, Vancouver, FR5PFP003(2009).

11. Y.Mori, International Workshop on FFAG Accelerators(FFAG2006), FNAL, Chicago, USA(2006).

12. S.Machida, Proc. of the International Workshop on FFAG Accelerators (FFAG05), KURRI, Osaka, Japan, 68(2005).

13. E.Yamakawa, T. Uesugi and Y.Mori, International Workshop on FFAG Accelerators(FFAG09), FNAL, Chicago, USA(2009).

14. S.Berg, International Workshop on FFAG Accelerators (FFAG05), KURRI, Osaka, Japan, 93(2005). 